\title{
Cost per response/remission in biologics available in Italy for the treatment of TNF- $\alpha$ inhibitors-naïve patients with ulcerative colitis
}

Global \& Regional Health Technology

Assessment

Volume 2019: I-9

(C) The Author(s) 2019

Article reuse guidelines: sagepub.com/journals-permissions DOI: 10.1 | $77 / 22842403$ | 8822434 journals.sagepub.com/home/grh @SAGE

\author{
Matteo Ruggeri and Francesca Romana Rolli
}

\begin{abstract}
Objective: This study compares the cost of a sustained response or remission (at 52 weeks' follow-up) across biologics approved in Italy for the treatment of moderately to severely active ulcerative colitis (UC), specifically among anti-TNF$\alpha$-naïve patients. The analysis is from the perspective of the national healthcare provider (SSN) for one year of treatment. Methods: Efficacy data about the induction of response/remission probabilities at 52 weeks and the number needed to treat (NNT) were derived from a network meta-analysis of randomized controlled clinical trials of the following drugs: infliximab (originator and biosimilar), adalimumab, golimumab and vedolizumab. It included the acquisition and administration costs of biologics, based on an activity-based costing analysis performed in 3 Italian centers of excellence for UC treatment.

Results: The costs per patient in sustained response at 52 weeks were, in increasing order: vedolizumab $€ 47,772$ $(95 \% \mathrm{Cl} € 29,869$ - €I0I,055), biosimilar infliximab €48,657 (95\% Cl €3I,488 - €95,523), golimumab (I00 mg every 4 weeks at maintenance) $€ 57,940$ (95\% Cl $€ 38,00-€ \mid 06,206)$, golimumab (50 mg every 4 weeks at maintenance) $€ 62,504$ (95\% Cl €39,976 - €I20,477), adalimumab €I0I,I8I (95\% Cl €49,635 - €422,334). The costs per patient in sustained remission at 52 weeks were: vedolizumab €86,220 (95\% Cl €47,0I5 - €206,652), biosimilar infliximab $€ 92,562(95 \% \mathrm{Cl} € 52,954-€ 203,619)$.

Conclusions: In patients with moderate to severe UC not previously treated with TNF- $\alpha$ inhibitors, treatment needed with vedolizumab to obtain a response or remission at 52 weeks of follow-up is less costly to the SSN compared with the other UC-approved biologics available in Italy.
\end{abstract}

\section{Keywords}

Cost-effectiveness, ulcerative colitis, biological therapies

Date received: 8 February 2018; revised: 17 September 2018; accepted: 25 September 2018

\section{Introduzione}

La colite ulcerosa (CU) è una malattia intestinale cronica caratterizzata dall'infiammazione diffusa della mucosa del retto e del colon, con un decorso variabile che prevede un'alternanza tra fasi di attività e periodi di remissione. I principali sintomi sono sangue nelle feci, diarrea mucoematica, dolori addominali, urgenza, tenesmo, talvolta accompagnati da sintomi sistemici come febbre e perdita di peso e da manifestazioni extra-intestinali. La gravità del quadro clinico è determinata dall'estensione e dal grado di infiammazione mucosale. ${ }^{1,2}$ Questa malattia è più comune nei paesi industrializzati e $\mathrm{i}$ tassi di incidenza in Europa (che hanno visto un incremento da 6,0/100.000 nel 1962 a 9,8/100.000 nel 2010) mostrano un gradiente nord $>$ sud e ovest>est, per quanto recentemente si sia notato un aumento delle diagnosi anche nei paesi tradizionalmente a

\footnotetext{
ALTEMS, Università Cattolica del Sacro Cuore, Rome, Italy

Corresponding author:

Prof. Matteo Ruggeri, ALTEMS, Università Cattolica del Sacro Cuore, Largo Francesco Vito I, Roma, 00I68, Italy.

Email: matteo.ruggeri@unicatt.it
} 
bassa incidenza. ${ }^{3,4}$ Per quanto riguarda l'Italia, i dati di incidenza, ottenuti in popolazioni e periodi diversi, variano da 5,2 a 14,5/100.000..$^{5-7}$ Un'indagine effettuata nel 2010 dalla Società Italiana di Medicina Generale ha mostrato che su 1.065.070 assistiti, 3.159 sono risultati affetti da CU in Italia (con una prevalenza quindi dello $0,30 \%$ ) e la fascia d'età più interessata è stata quella tra 65 e 75 anni. ${ }^{8}$ Nello stesso anno, le visite specialistiche per la $\mathrm{CU}$ sono state quasi $3.000 .^{8}$

L'impatto della CU sulla vita delle persone affette è notevole, con interferenze sia nel lavoro (riportate dal $66 \%$ dei pazienti) sia nelle attività di svago $(73 \%) .{ }^{1} \mathrm{I}$ sintomi della malattia, l'età spesso giovane dei pazienti, l'imprevedibilità delle recidive e lo stigma spesso associato alle patologie croniche sono i fattori che contribuiscono maggiormente al deterioramento della qualità della vita (QOL) dei pazienti con $\mathrm{CU}$ ed è stata rilevata una correlazione tra il grado di severità della malattia e la riduzione nella QOL. ${ }^{9}, 10$ Come documentato da numerosi studi, la CU comporta costi significativi per la società, sia diretti (visite, accertamenti diagnostici, terapie e ricoveri ospedalieri) sia indiretti (assenteismo, perdita di produttività). ${ }^{9}, 11,12$

Tale patologia non è curabile e prevede un approccio terapeutico di tipo medico $\mathrm{o}$, nei casi più gravi, chirurgico. Lo scopo della terapia si è evoluto, negli ultimi anni, dal controllo dei sintomi nella fase acuta e l'induzione della remissione clinica, a obiettivi più stringenti, quali mantenere la remissione libera da steroidi, prevenire l'ospedalizzazione e gli interventi chirurgici, ottenere la guarigione mucosale, migliorare la QOL ed evitare la disabilità (13). Il trattamento deve essere adattato alle attività della malattia (lieve, moderata, grave) e all'entità del coinvolgimento del colon (proctite, colite sinistra o pancolite). ${ }^{13}$ La terapia medica convenzionale, che comprende aminosalicilati, corticosteroidi e immunosoppressori, spesso non è in grado di indurre o mantenere una risposta. L'avvento dei farmaci biologici ha modificato sostanzialmente la gestione della $\mathrm{CU}$, incrementando $\mathrm{i}$ tassi di risposta e remissione rispetto ai trattamenti convenzionali nei pazienti con attività di malattia da moderata a grave. ${ }^{14-18}$

I primi farmaci biologici approvati in Italia per i pazienti con CU sono stati gli inibitori del TNF- $\alpha$, infliximab, adalimumab e golimumab. Più recentemente è stato introdotto vedolizumab, un antagonista del recettore dell'integrina $\alpha_{4} \beta_{7}$ umana ad attività selettiva intestinale. Questo farmaco ha dimostrato, nello studio GEMINI 1, un'efficacia significativamente superiore al placebo nella terapia di induzione e di mantenimento, sia in pazienti non precedentemente esposti al trattamento con inibitori del TNF- $\alpha$ sia in quelli che non avevano risposto a tale trattamento. ${ }^{19}$ Vedolizumab è stato approvato anche in Italia ed è indicato per il trattamento dei pazienti adulti con $\mathrm{CU}$ attiva da moderata a grave che hanno mostrato una risposta clinica inadeguata, hanno perso la risposta o sono risultati intolleranti alla terapia convenzionale o alla somministrazione di un inibitore del TNF- $\alpha$. Ai fini della razionalizzazione della spesa sanitaria, è importante confrontare la costo-efficacia dei vari farmaci biologici disponibili per il trattamento della CU. Tale analisi è, però, resa difficile dall'assenza di dati di efficacia derivanti da confronti diretti tra i farmaci biologici considerati. Per ovviare a questa criticità, sono state effettuate revisioni sistematiche mediante metanalisi a rete (network metaanalysis) che hanno stimato indirettamente, con metodi bayesiani, l'efficacia e la tollerabilità comparativa delle varie terapie innovative. ${ }^{20-22}$

In questo studio, si sono confrontati i costi associati ai farmaci biologici approvati in Italia (infliximab originatore, infliximab biosimilare, adalimumab, golimumab e vedolizumab), necessari per ottenere una risposta sostenuta o la remissione a 52 settimane, in pazienti con $\mathrm{CU}$ ad attività da moderata a grave, non precedentemente trattati con inibitori del TNF- $\alpha$. Per tale valutazione, condotta nella prospettiva degli ospedali afferenti al Sistema Sanitario Nazionale (SSN), sono stati utilizzati i dati di efficacia stimati attraverso una metanalisi a rete.

\section{Metodi}

Questa analisi economica è stata condotta applicando un approccio misto che si avvale dell'utilizzo sia di dati primari che secondari. Infatti, i costi di acquisto dei farmaci e di somministrazione sono stati ricavati tramite un'indagine sul campo, mentre i dati di efficacia sono stati ottenuti dalla letteratura. L'obiettivo è di misurare e valorizzare il consumo di risorse associato alla risposta o alla remissione clinica, in pazienti con $\mathrm{CU}$, naïve agli inibitori del TNF- $\alpha$, alla settimana 52 di trattamento con farmaci biologici.

\section{Raccolta dei dati}

I dati di efficacia utilizzati ai fini della presente analisi derivano da una metanalisi a rete, in corso di pubblicazione, condotta al fine di attuare confronti indiretti dei trial clinici relativi alle terapie biologiche disponibili in Italia per il trattamento dei pazienti adulti affetti da $\mathrm{CU}$, in fase attiva, di grado da moderato a grave. ${ }^{22}$ La metanalisi a rete si alimenta con i dati di efficacia ottenuti dalla letteratura grazie ad una revisione sistematica, condotta secondo le impostazioni per modelli lineari generalizzati proposte da Dias et al. ${ }^{23}$ Nella metanalisi, sono stati inclusi gli studi clinici randomizzati e controllati con placebo relativi a infliximab (IFX) ${ }^{14}$ adalimumab (ADA) ${ }^{24}$ golimumab $(\mathrm{GOL})^{16,17}$ e vedolizumab (VDZ) ${ }^{18}$ nella popolazione in oggetto. Tutti gli studi valutavano la risposta clinica e la remissione clinica. La risposta clinica è definita come una riduzione di minimo 3 punti del punteggio Mayo e di 
Tabella I. Probabilità di risposta sostenuta/remissione a 52 settimane e numero necessario di soggetti da trattare (NNT) per ottenere una risposta sostenuta/remissione a 52 settimane attraverso l'utilizzo di farmaci biologici in pazienti anti-TNF- $\alpha$-naïve con $\mathrm{CU}$ ad attività da moderata a grave.

\begin{tabular}{|c|c|c|c|c|}
\hline \multicolumn{5}{|l|}{ RISPOSTA } \\
\hline & \multicolumn{2}{|c|}{$\begin{array}{l}\text { Probabilità di risposta sostenuta a } 52 \\
\text { settimane }\end{array}$} & \multicolumn{2}{|c|}{$\begin{array}{l}\text { NNT per risposta sostenuta a } 52 \\
\text { settimane }\end{array}$} \\
\hline & Stima & IC $95 \%$ & Stima & IC $95 \%$ \\
\hline Placebo & 0,12 & $0,09-0,15$ & Rif & \\
\hline Infliximab 5 mg/kgrif $*$ & 0,34 & $0,23-0,47$ & 4,5 & $2,9-8,9$ \\
\hline Adalimumab $160 / 80 / 40 \mathrm{mg}^{\text {rif } *}$ & 0,22 & $0,|4-0,3|$ & 10,2 & $5,0-45,4$ \\
\hline Golimumab $200 / 100 / 100$ mgrif $*$ & 0,31 & $0,22-0,4 I$ & 5,2 & $3,4-9,5$ \\
\hline Vedolizumab 300 mgrif $*$ & 0,40 & $0,26-0,59$ & 3,6 & $2,2-7,5$ \\
\hline \multicolumn{5}{|l|}{ REMISSIONE } \\
\hline & \multicolumn{2}{|c|}{$\begin{array}{l}\text { Probabilità di remissione a } 52 \\
\text { settimane }\end{array}$} & \multicolumn{2}{|c|}{ NNT per remissione a 52 settimane } \\
\hline & Stima & IC $95 \%$ & Stima & IC 95\% \\
\hline Placebo & 0,04 & $0,03-0,05$ & Rif & \\
\hline Infliximab 5 mg/kgrif $*$ & 0,16 & $0,09-0,24$ & 8,5 & $4,8-18,9$ \\
\hline Adalimumab $160 / 80 / 40 \mathrm{mg}^{\text {rif* }}$ & 0,08 & $0,04-0,14$ & 22,4 & $9,7-108,8$ \\
\hline Golimumab $200 / 100 / 100$ mgrif $*$ & 0,13 & $0,08-0,21$ & 10,2 & $6,0-20,7$ \\
\hline Vedolizumab $300 \mathrm{mg}^{\text {rif } *}$ & 0,19 & $0,11-0,32$ & 6,5 & $3,5-15,4$ \\
\hline
\end{tabular}

IC 95\%: Intervalli di confidenza al 95\%; NNT: number-needed-to-treat.

*Vedere la Tabella 2 per i dettagli sui dosaggi.

almeno il 30\% rispetto al basale (con una diminuzione del punteggio per il sanguinamento rettale di almeno 1 punto) o un punteggio assoluto per il sanguinamento rettale non superiore a 1. Per remissione clinica si intende, invece, un punteggio Mayo complessivamente minore o uguale a 2, con nessun sotto punteggio superiore a 1. Il Mayo Score è un metodo di misurazione tra $\mathrm{i}$ più utilizzati nelle valutazioni di pazienti con problemi gastroenterologici. Esso, attraverso 12 valori, rileva l'attività della malattia mediante la valutazione di quattro dimensioni, ossia la frequenza di evacuazione, il sanguinamento rettale, i risultati endoscopici e il giudizio complessivo del medico. A ogni fattore è attribuito un valore da 0 (valore normale o patologia inattiva) a 3 (attività grave). Un punteggio complessivo elevato corrisponde ad uno stadio avanzato della patologia.

Dalla metanalisi sono stati ricavati, in particolare, $\mathrm{i}$ dati di efficacia osservati a 52 settimane e relativi alla probabilità di ottenere una risposta clinica sostenuta o una remissione registrata nella popolazione non precedentemente trattata con anti-TNF- $\alpha$. È stato quindi calcolato il numero di pazienti da trattare (NNT) per ottenere un paziente in risposta sostenuta/remissione a 52 settimane, come il reciproco del tasso di risposta incrementale vs placebo per ogni trattamento. ${ }^{25}$ Ricordiamo che un trattamento è tanto più efficace quanto più basso è l'NNT.
I dati di efficacia descritti e utilizzati ai fini dello studio sono riportati nella Tabella 1.

\section{Analisi dei dati e calcolo dei costi}

I dati di costo utilizzati includono sia l'acquisto dei farmaci biologici sia i costi di somministrazione. ${ }^{26}$ Per quanto riguarda i costi di acquisto, il calcolo è stato eseguito considerando la posologia standard riportata nei riassunti delle caratteristiche di prodotto (RCP) delle terapie biotecnologhe autorizzate per il trattamento della CU moderata-severa. ${ }^{27-34}$ Come riportato nella Tabella 2, i costi di acquisizione dei farmaci sono stati calcolati in base a quanto indicato nelle RCP e in base ai prezzi pubblicati sulla Gazzetta Ufficiale (comprensivi degli sconti $5 \%+5 \%$ e non comprensivi di sconti non visibili).

I costi di somministrazione, invece, comprendono l'assistenza sanitaria necessaria per dispensare e somministrare le terapie individuate al paziente (visite mediche, assistenza infermieristica, procedure amministrative e materiali di consumo). Nello specifico, i costi utilizzati sono stati calcolati utilizzando il metodo dell'activity-based costing (ABC). ${ }^{35,36}$ Attraverso l'utilizzo di tale approccio è possibile determinare il costo di ciascuna attività che compone il processo e dunque attribuire tale costo all'output finale. L'analisi è stata 
Tabella 2. Costo unitario di acquisto e somministrazione dei farmaci biologici e numero di unità utilizzate durante la fase di induzione (per paziente trattato).

\begin{tabular}{|c|c|c|c|c|c|}
\hline Intervento & $\begin{array}{l}\text { Descrizione (dosaggio, } \\
\text { somministrazione e posologia } \\
\text { all'induzione) }\end{array}$ & $\begin{array}{l}\text { Farmaco } \\
\text { (quantità) o } \\
\text { somministrazione }\end{array}$ & $\begin{array}{l}\text { Costo per } \\
\text { unità }(€)^{*}\end{array}$ & $\begin{array}{l}\text { No. unità } \\
\text { utilizzate** }\end{array}$ & $\begin{array}{l}\text { Costo totale } \\
\text { per l'induzione } \\
(€)\end{array}$ \\
\hline \multirow[t]{2}{*}{ Infliximab biosimilare } & $5 \mathrm{mg} / \mathrm{kg}$ settimane $0,2,6$. Periodo di & Fiala 100 mg & 386 & 12 & \multirow[t]{2}{*}{5.206} \\
\hline & $\begin{array}{l}8 \text { settimane per valutazione risposta, } \\
\text { assumendo paziente di } 75 \mathrm{~kg} \text { e sprechi }\end{array}$ & Somministrazione & 190 & 3 & \\
\hline Adalimumab & \multirow{2}{*}{$\begin{array}{l}\text { Inizialmente } 160 \mathrm{mg}, 80 \mathrm{mg} \text { dopo } 2 \\
\text { settimane, } 40 \mathrm{mg} \text { a settimane alterne. } \\
\text { Interruzione se mancata risposta a } 8 \\
\text { settimane }\end{array}$} & $40 \mathrm{mg}$ iniettabile & 482 & 8 & \multirow[t]{2}{*}{4.332} \\
\hline $160 / 80 / 40 \mathrm{mg}$ & & Somministrazione & 119 & 4 & \\
\hline Golimumab & \multirow{2}{*}{$\begin{array}{l}\text { Settimana 0, } 200 \mathrm{mg} \text { ( } 2 \text { iniezioni); } \\
\text { settimana } 2,100 \mathrm{mg} \text { ( } 1 \text { iniezione). } \\
\text { NOTA: regime di mantenimento inizia } \\
\text { a } 6 \text { settimane (da trial) }\end{array}$} & 100 mg iniettabile & 1.044 & 3 & \multirow[t]{2}{*}{3.488} \\
\hline $200 / 100 / 100 \mathrm{mg}$ & & Somministrazione & 119 & 3 & \\
\hline \multirow[t]{2}{*}{ Vedolizumab 300 mg } & \multirow{2}{*}{$\begin{array}{l}300 \text { mg infusione endovenosa a } \\
\text { settimane } 0,2 \text {, e } 6\end{array}$} & Fiala 300 mg & 2.036 & 3 & \multirow[t]{2}{*}{6.560} \\
\hline & & Somministrazione & $|5|$ & 3 & \\
\hline
\end{tabular}

Costi di somministrazione del farmaco derivano da un'analisi di activity-based costing condotta in tre centri di eccellenza italiani.

*Riferimenti 28, 30, 32 e 34.

***Riferimenti $27,29,31$ e 33

condotta in tre centri italiani di eccellenza nel trattamento della CU: Policlinico Tor Vergata, Policlinico Universitario Agostino Gemelli e Azienda Ospedaliera San CamilloForlanini, Roma. ${ }^{26}$ Ai fini dell'analisi di ABC, è stato chiesto a un panel di clinici di stimare il tempo impiegato da ciascun professionista sanitario per somministrare il farmaco biologico. I costi di somministrazione relativi a ciascun trattamento biologico sono stati ricavati moltiplicando il tempo impiegato per la dispensazione/ somministrazione del farmaco per il salario orario dei professionisti sanitari coinvolti. ${ }^{26}$

\section{Calcolo del costo per NNT}

Il costo totale per paziente con risposta o remissione clinica (rispetto al placebo) è stato calcolato generando per ogni NNT attribuito ai trattamenti una distribuzione Beta che tenesse conto del NNT medio e dell'intervallo di confidenza. Il costo medio sostenuto dall'erogatore dei assistenza sanitaria è stato stimato considerando il cinquantesimo percentile della distribuzione ottenuta.

\section{Analisi di sensibilità}

Al fine di esplorare l'incertezza dei risultati del modello, nonché validarne la robustezza, è stata condotta un'analisi di sensibilità probabilistica sul costo per risposta sostenuta e remissione ottenuta con i trattamenti considerati nello studio, lanciando con il metodo Montecarlo 1000 simulazioni. L'analisi Montecarlo considera i dati di efficacia ottenuti da una media delle metanalisi a rete considerate e $\mathrm{i}$ costi ottenuti dai tre centri di eccellenza. Per modellare tutti i parametri considerati nell'analisi, si è scelto di utilizzare delle variabili normali. In particolare, per quanto riguarda $i$ dati di efficacia, a partire dagli intervalli di confidenza, sono state costruite delle variabili casuali normali standardizzate che modellassero una variabilità compresa fra 0 e 1 . È stata inoltre condotta un'analisi di sensibilità univariata al fine di osservare le variazioni del costo per risposta sostenuta e del costo per remissione sostenuta conseguenti a una variazione del prezzo dei trattamenti del $10 \%$, del $20 \%$ e del $30 \%$.

\section{Risultati}

Le Tabelle 2 e 3 mostrano rispettivamente il costo unitario per l'acquisto e la somministrazione del farmaco e il numero di unità utilizzate durante le fasi di induzione e di mantenimento.

Il costo per paziente con risposta sostenuta a 52 settimane di follow-up per vedolizumab è risultato pari a $€ 47.772$ (95\% CI €29.869 - €101.055) ed è l'importo più basso se confrontato con i valori ottenuti per gli altri farmaci biologici. Infatti, a seguire, in ordine crescente, vi sono infliximab biosimilare [€48.657 (95\% CI €31.488 $€ 95.523)$ ], golimumab al dosaggio di $100 \mathrm{mg}$ e di $50 \mathrm{mg}$ ogni 4 settimane di mantenimento [€57.940 (95\% CI $€ 38.001$ - €106.206) ed €62.504 (95\% CI €39.976 $€ 120.477)$, rispettivamente] e adalimumab [€101.181 (95\% CI €49.635 - €422.334)]. Come osservabile dalla Figura 1, tutti i trattamenti rimangono intorno ad un range di variazione che oscilla fra $€ 47.000$ ed $€ 63.000$. Unica eccezione è rappresentata da adalimumab, il quale non solo oltrepassa di gran lunga l'intervallo sopra citato, ma si discosta da esso di circa $€ 40.000$. 
Tabella 3. Costo unitario di acquisto e somministrazione dei farmaci biologici e numero di unità utilizzate durante la fase di mantenimento (per paziente trattato).

\begin{tabular}{|c|c|c|c|c|c|}
\hline Intervento & $\begin{array}{l}\text { Descrizione (dosaggio, } \\
\text { somministrazione e posologia } \\
\text { nel mantenimento) }\end{array}$ & $\begin{array}{l}\text { Farmaco } \\
\text { (quantità) o } \\
\text { somministrazione }\end{array}$ & $\begin{array}{l}\text { Costo per } \\
\text { unità }(€)^{*}\end{array}$ & $\begin{array}{l}\text { No. unità } \\
\text { utilizzate }^{* *}\end{array}$ & $\begin{array}{l}\text { Costo totale per il } \\
\text { mantenimento }(€)\end{array}$ \\
\hline \multirow[t]{2}{*}{ Infliximab biosimilare } & \multirow{2}{*}{$\begin{array}{l}5 \mathrm{mg} / \mathrm{kg} \text { ogni } 8 \text { settimane, } \\
\text { assumendo paziente di } 70 \mathrm{~kg} \\
\text { e sprechi }\end{array}$} & Fiala $100 \mathrm{mg}$ & 386 & 20 & \multirow[t]{2}{*}{8.413} \\
\hline & & Somministrazione & 137 & 5 & \\
\hline \multirow[t]{2}{*}{ Adalimumab $160 / 80 / 40 \mathrm{mg}$} & \multirow[t]{2}{*}{$40 \mathrm{mg}$ a settimane alterne } & $40 \mathrm{mg}$ iniettabile & 482 & 22 & \multirow[t]{2}{*}{ I I.427 } \\
\hline & & Somministrazione & 63 & 13 & \\
\hline \multirow{2}{*}{ Golimumab $200 / 100 / 100 \mathrm{mg}$} & \multirow[t]{2}{*}{ I00 mg ogni 4 settimane } & $100 \mathrm{mg}$ iniettabile & 1.044 & 12 & \multirow[t]{2}{*}{13.034} \\
\hline & & Somministrazione & 63 & 8 & \\
\hline \multirow[t]{2}{*}{ Vedolizumab 300 mg } & \multirow[t]{2}{*}{300 mg ogni 8 settimane } & Fiala $300 \mathrm{mg}$ & 2.036 & 5 & \multirow[t]{2}{*}{10.670} \\
\hline & & Somministrazione & 98 & 5 & \\
\hline
\end{tabular}

Costi di somministrazione del farmaco derivano da un'analisi di activity-based costing condotta in tre centri di eccellenza italiani. *Riferimenti 28, 30, 32 e 34.

**Riferimenti $27,29,31$ e 33 .

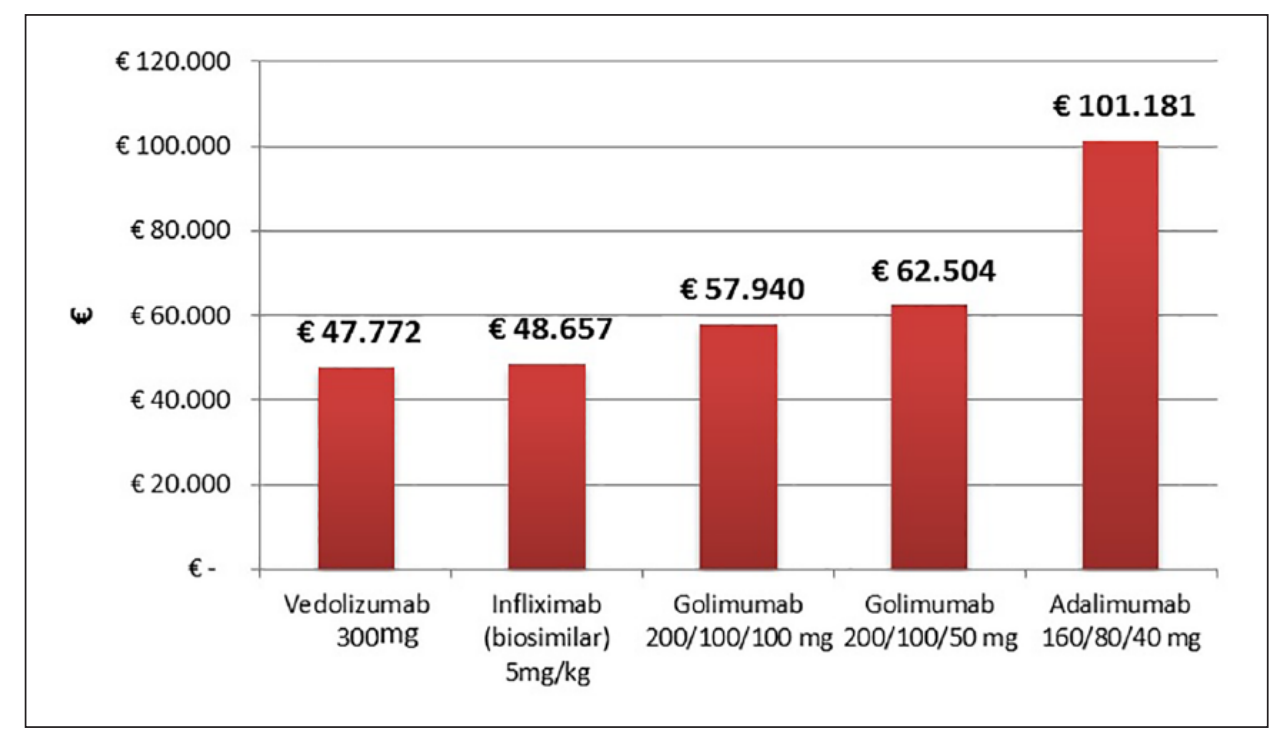

Figura I. Costo per paziente con risposta clinica sostenuta a 52 settimane tra pazienti anti-TNF- $\alpha$-naïve con colite ulcerosa in fase attiva di grado da moderato a grave.

Secondo l'analisi di sensibilità probabilistica, i costi associati a vedolizumab per ottenere una risposta clinica sostenuta non hanno superato comunque nella totalità delle simulazioni $€ 90.000$, mentre adalimumab si è confermato l'alternativa terapeutica maggiormente onerosa (Figura 2). Allo stesso modo, il costo per paziente in remissione sostenuta a 52 settimane di follow-up per vedolizumab è risultato il più basso tra tutti i biologici considerati $[€ 86.220$ (95\% CI €47.015 - €206.652)], seguito da infliximab biosimilare [€92.562 (95\% CI €52.954 - €203.619)], golimumab 200/100/100 mg (€115.000), golimumab 200/100/50 mg (€126.168) e adalimumab (€221.762). La Figura 3 mostra come adalimumab è il trattamento che, in termini di costo per NNT, è l'alternativa che assorbe maggiori risorse.
Come si evince dalla Figura 4, la remissione comporta generalmente costi più elevati. Tuttavia, anche in questa fase, l'analisi di sensibilità ha mostrato dati conformi a quanto emerso per la risposta clinica. Il trattamento con vedolizumab non ha superato nella totalità delle simulazioni $€ 180.000$, differentemente da adalimumab, all'estremo opposto, per il quale il valore massimo riportato è pari a $€ 500.000$.

Infine, la Tabella 4 riporta i risultati dell'analisi di sensibilità univariata che tiene conto della variazione dei prezzi dei trattamenti del $10 \%$, del $20 \%$ e del $30 \%$. Dai risultati si evince che, sia nel caso del costo per risposta sostenuta sia nel caso del costo per remissione, solo nel confronto con infliximab scontato al 20\% (o 30\%), vedolizumab risulterebbe meno vantaggioso qualora 


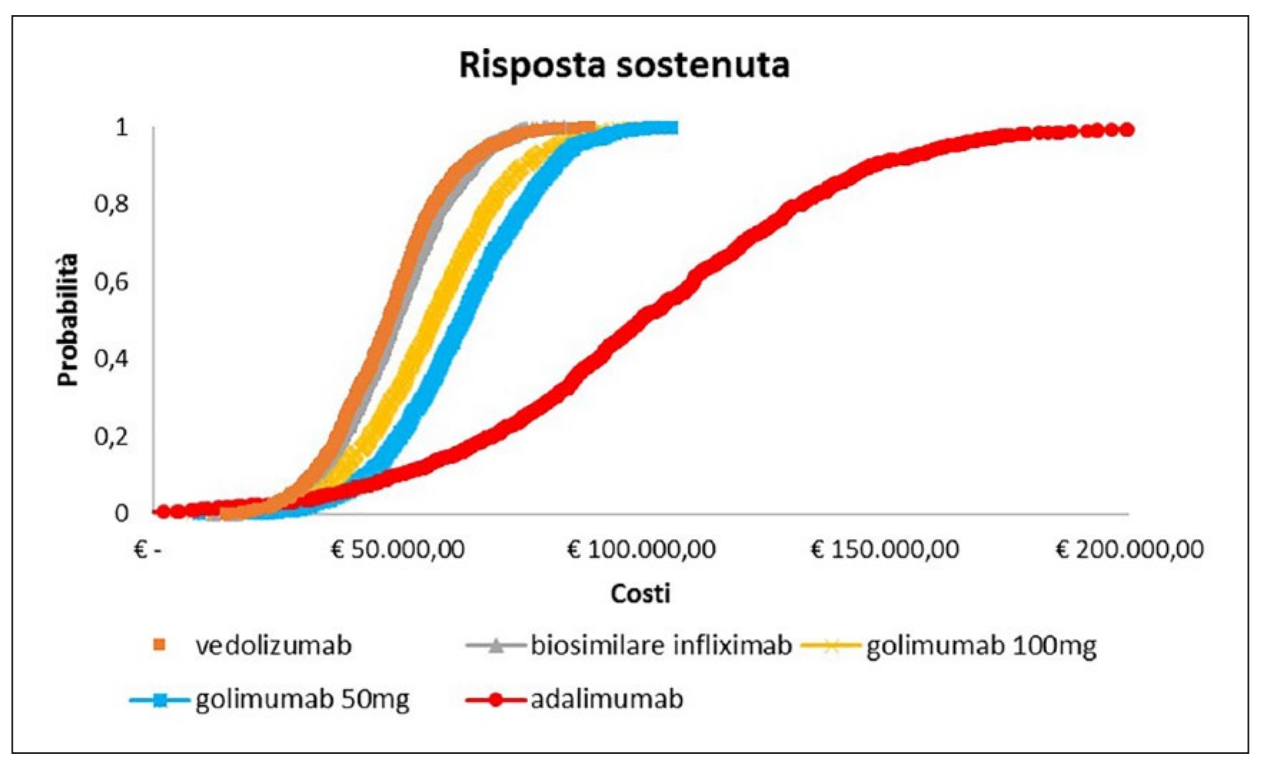

Figura 2. Curve probabilistiche dei costi per paziente con risposta clinica sostenuta a 52 settimane (pazienti anti-TNF- $\alpha$-naïve con colite ulcerosa in fase attiva di grado da moderato a grave).

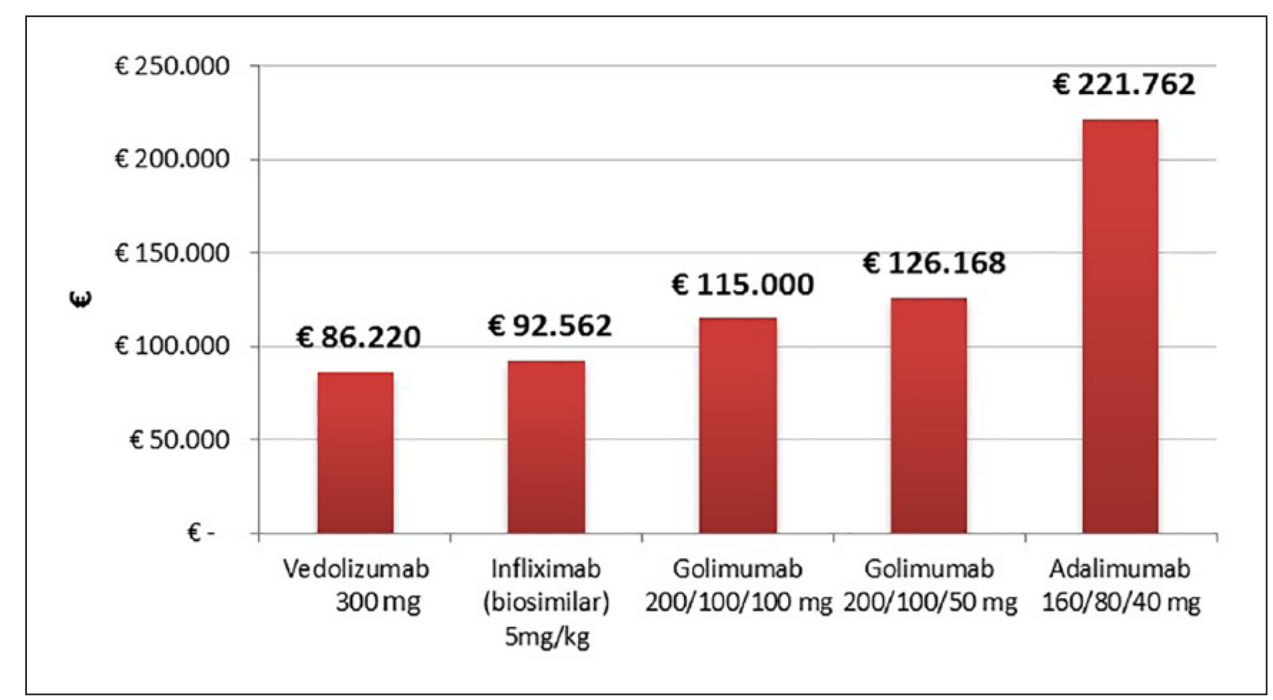

Figura 3. Costo per paziente con remissione clinica sostenuta a 52 settimane tra pazienti anti-TNF- $\alpha$-naïve con colite ulcerosa in fase attiva di grado da moderato a grave.

scontato solo al $10 \%$. Negli altri confronti anche uno sconto sui prezzi dei comparator del $30 \%$ non si tradurrebbe mai in un costo inferiore a quello di vedolizumab.

\section{Discussione}

Il presente studio ha mostrato come, tra i farmaci biologici approvati in Italia per il trattamento della CU di grado da moderato a severo, per ottenere una risposta sostenuta o una remissione a 52 settimane di terapia, in pazienti naïve agli anti-TNF- $\alpha$, vedolizumab può rappresentare l'opzione meno onerosa per il SSN. Gli altri farmaci biologici considerati si sono rivelati più costosi sia per la risposta clinica sia per la remissione: in ordine crescente, infliximab biosimilare, golimumab al dosaggio di $100 \mathrm{mg}$ e $50 \mathrm{mg}$, e adalimumab. Adalimumab, in particolare, è risultato il farmaco più oneroso $(€ 101.181$ per risposta sostenuta e $€ 221.762$ per remissione sostenuta), superando con ampio margine il range rappresentato dai costi degli altri biologici. Al fine di esplorare l'incertezza dei risultati del modello, nonché validarne la robustezza, è stata condotta un'analisi di sensibilità probabilistica a partire dai costi dei trattamenti considerati nello studio, attraverso la quale, lanciando con il metodo Montecarlo 1000 simulazioni, è emerso come nella totalità delle stesse (e quindi nella totalità della casistica a livello di probabilità) 


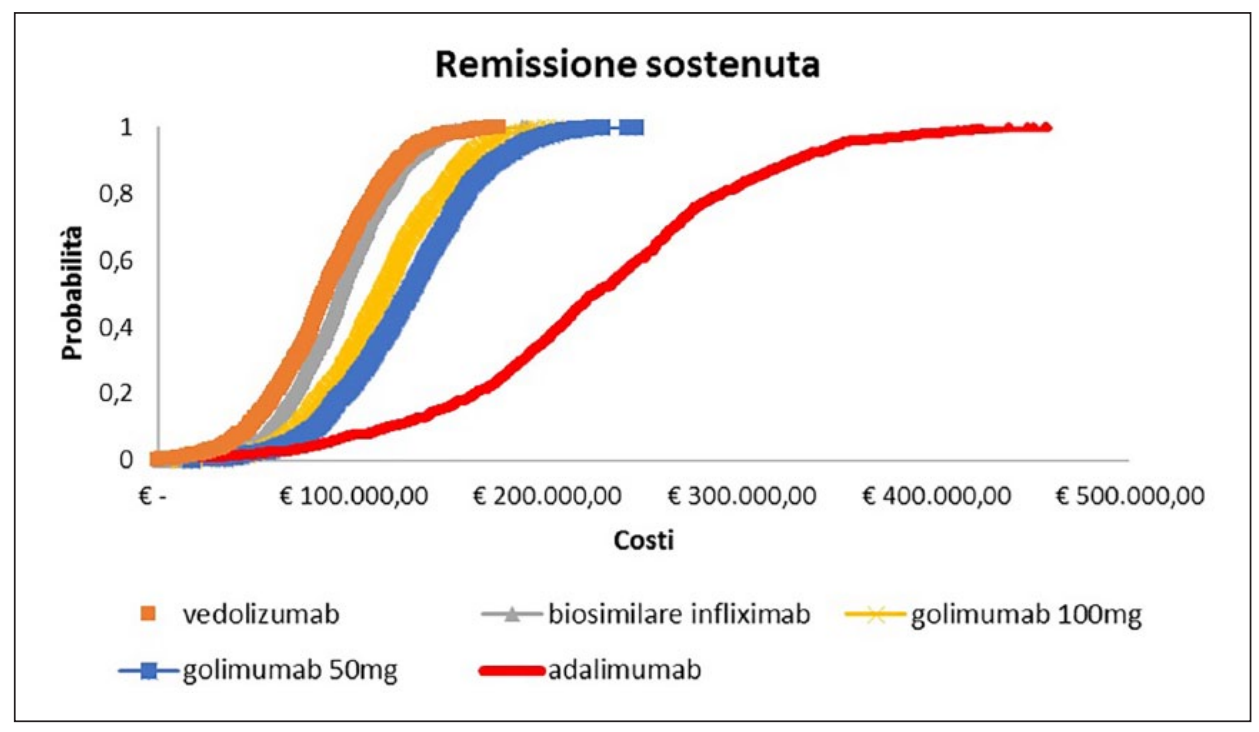

Figura 4. Curve probabilistiche dei costi per paziente con remissione clinica sostenuta a 52 settimane (pazienti anti-TNF- $\alpha$-naïve con colite ulcerosa in fase attiva di grado da moderato a grave).

Tabella 4. Analisi di sensibilità univariata sul prezzo di acquisto. Ipotesi scontistica: $10 \%, 20 \%$ e $30 \%$.

\begin{tabular}{lllll}
\hline Sconto & Vedolizumab & Infliximab & Golimumab* & Adalimumab \\
\cline { 2 - 5 } & costo per risposta sostenuta & & & \\
\hline $10 \%$ & $€ 44.582,00$ & $€ 46.872,00$ & $€ 56.841,00$ & $€ 90.217,00$ \\
$20 \%$ & $€ 39.471,00$ & $€ 41.528,00$ & $€ 47.124,00$ & $€ 78.541,00$ \\
$30 \%$ & $€ 35.681,00$ & $€ 37.562,00$ & $€ 40.561,00$ & $€ 71.426,00$ \\
\hline & costo per remissione sostenuta & & $€ 195.623,00$ \\
\hline $10 \%$ & $€ 80.216,00$ & $€ 85.201,00$ & $€ 106.524,00$ & $€ 182.305,00$ \\
$20 \%$ & $€ 72.548,00$ & $€ 77.526,00$ & $€ 99.542,00$ & $€ 167.357,00$ \\
$30 \%$ & $€ 63.411,00$ & $€ 68.542,00$ & $€ 91.247,00$ &
\end{tabular}

*Media del costo terapia sui due dosaggi disponibili (50 mg o $100 \mathrm{mg}$ ogni 4 settimane).

il costo di vedolizumab non superi comunque $€ 90.000$. Differentemente, adalimumab rappresenta l'alternativa terapeutica maggiormente onerosa. In termini di costoefficacia, tale risultato sembra confermato anche da un recente studio condotto in UK in una popolazione di pazienti con colite ulcerosa moderata-severa: vedolizumab è risultato essere un'alternativa costo-efficace rispetto agli anti-TNF. ${ }^{37}$

In assenza di studi clinici di confronto diretto tra $\mathrm{i}$ farmaci biologici esaminati, i dati di efficacia che abbiamo utilizzato per modellare i costi derivano da un confronto indiretto realizzato mediante una network metanalisi. Questa metodologia, nonostante i limiti necessariamente imposti dal raggruppamento di dati provenienti da trial clinici diversi (sia pure simili per struttura, criteri di arruolamento e outcome) è ampiamente utilizzata nei più recenti studi di farmacoeconomia che analizzano la CU. ${ }^{37,38}$ Tuttavia, le potenziali distorsioni dei risultati, indotte dall'eterogeneità delle popolazioni considerate $\mathrm{e}$ dal confronto fra studi disegnati con criteri di randomizzazione e protocolli potenzialmente differenti, rende opportuno porsi delle domande sulla effettiva verificabilità delle differenze di efficacia stimate. La domanda principale riguarda il metodo di stima basato su un modello lineare generalizzato che potenzialmente rilassa l'assunzione sull'omogeneità della popolazione inclusa nello studio. Questo implica tuttavia una assunzione "a monte" sulla struttura da dare ai residui e quindi sulla distribuzione "a priori" da assegnare al dato di efficacia nel caso in cui l'analisi segua l'approccio bayesiano. Gli autori hanno condotto, sulla base dei risultati dati dalla letteratura, alcune simulazioni utilizzando distribuzioni diverse da quelle utilizzate negli studi pubblicati, ottenendo risultati sensibilmente differenti ma che, tuttavia non cambiavano l'esito dell'analisi che assegnava a vedolizumab la probabilità di risposta e remissione più alta rispetto agli altri trattamenti. Resta inteso che l'utilizzo di tali strumenti deve essere considerato utile solo se inteso 
come integrativo e non sostitutivo rispetto alle evidenze dei clinical trials. Per quanto riguarda il calcolo dei costi di somministrazione dei vari farmaci, abbiamo condotto un'analisi di activity-based costing, avvalendoci delle stime fornite da un panel di clinici operanti in tre centri clinici di eccellenza nel trattamento della CU. Questa metodologia di calcolo dell' $\mathrm{ABC}$ attribuisce i costi delle risorse alle attività svolte per la produzione del bene o del servizio, superando totalmente la logica di costi fissi e variabili. ${ }^{35,36}$

Dal momento che i pazienti precedentemente trattati con biologici e quelli naïve a questi farmaci rappresentano verosimilmente due popolazioni cliniche diverse, ${ }^{21} \mathrm{ci}$ siamo attenuti ai dati relativi ai pazienti non precedentemente trattati con inibitori del TNF- $\alpha$. Le nostre conclusioni, pertanto, riguardano solo questo sottogruppo di pazienti con $\mathrm{CU}$.

Ricordiamo, inoltre, che sono stati inclusi nell'analisi solo i costi diretti di acquisizione e somministrazione dei farmaci biologici, mentre non sono stati presi in considerazione i costi relativi a terapie concomitanti, eventuali terapie per eventi avversi, ospedalizzazione, chirurgia o monitoraggio diagnostico. D'altra parte, l'orizzonte temporale dell'analisi (52 settimane) non avrebbe consentito di ottenere risultati robusti in termini di riduzione di altri costi sanitari diretti. Inoltre, la prospettiva utilizzata, cioè quella degli ospedali del SSN, non prende in considerazione eventuali costi (es.: DRG) per ospedalizzazione che sarebbero a carico del SSN. Includere tali costi secondo la prospettiva ospedaliera avrebbe significato applicare una metodologia $\mathrm{ABC}$ anche per stimare il costo per l'ospedale di eventuali complicanze, il che avrebbe reso lo studio oltremodo complesso, aggiungendo informazione poco rilevante rispetto agli obiettivi. Infine, come evidenziato da uno studio sull'evoluzione dei costi sostenuti per le malattie infiammatorie croniche intestinali nei Paesi Bassi a partire dal 2011, l'uso dei farmaci biologici sta assumendo un'importanza crescente come driver di costi: nel corso dei 2 anni di follow-up, a fronte di una spesa sanitaria totale per la CU sostanzialmente invariata, la frazione rappresentata dal costo della terapia con biologici era aumentata dal $31 \%$ al $39 \%$, mentre era diminuita dal $22 \%$ al $15 \%$ quella correlata ai costi di ospedalizzazione. ${ }^{39} \mathrm{Ai}$ fini delle esigenze di contenimento della spesa sanitaria da parte del SSN, appare quindi evidente la necessità di decisioni oculate nella scelta terapeutica, basate su principi di costo-efficacia.

Nella nostra analisi di confronto tra gli agenti biologici prescrivibili in Italia per il trattamento della $\mathrm{CU}$, vedolizumab appare la scelta più razionale nei pazienti non precedentemente trattati con inibitori del TNF- $\alpha$.

Occorre sottolineare che i risultati di efficacia e di costo disponibili sono relativi a un orizzonte temporale di breve periodo (un anno, pari a 52 settimane) seppure la malattia (colite ulcerosa) sia cronica. Ciò spiega l'apparente alto valore degli ICER stimati che, laddove venisse considerato un modello pluriennale, sarebbero sensibilmente ridotti. È inoltre da considerare che il costo per risposta sostenuta e quello per remissione ottenuta, non esauriscono l'intero valore dei trattamenti che, oltre ad evitare ricadute, ospedalizzazioni e ulteriori complicanze, hanno l'obiettivo finale di migliorare i guadagni di salute. Questo, avrebbe reso necessaria la costruzione di un modello Markoviano pluriennale e l'utilizzo di un rapporto incrementale di costo-efficacia (ICER), basato sul costo per anno di vita aggiustato per la qualità di vita (QALY) guadagnato che sarà tuttavia implementato come ideale prosecuzione del presente studio. Ė auspicabile quindi che queste conclusioni siano confermate e rafforzate con nuove evidenze ed eventualmente supportate da risultati di costo-efficacia real-life nel contesto sanitario italiano, considerando orizzonti temporali più ampi.

\section{Conclusioni}

Nell'ambito dei farmaci biologici approvati in Italia per il trattamento della $\mathrm{CU}$ da moderata a grave, prendendo in esame la risposta o la remissione a un anno in pazienti non precedentemente trattati con inibitori del TNF- $\alpha$, la terapia con vedolizumab è l'opzione meno onerosa per il SSN. Ulteriori analisi basate sulle scontistiche da gara pubblica sarebbero auspicabili per approfondire le tendenze riscontrate nel presente studio.

\section{Declaration of Conflicting Interest}

The authors declare that there is no conflict of interest.

\section{Funding}

This study benefited from an Investigator-Initiated Sponsored Research grant from Takeda Italia.

\section{References}

1. Dignass A, Eliakim R, Magro F, et al. Second European evidence-based consensus on the diagnosis and management of ulcerative colitis part 1: definitions and diagnosis. $J J$ Crohns Colitis 2012; 6(10): 965-990.

2. Danese $\mathrm{S}$ and Fiocchi C. Ulcerative colitis. $N$ Engl J Med. 2011; 365(18):1713-1725.

3. Burisch $J$ and Munkholm P. The epidemiology of inflammatory bowel disease. Scand J Gastroenterol 2015; 50(8): 942-951.

4. Lakatos PL. Recent trends in the epidemiology of inflammatory bowel diseases: up or down? World $J$ Gastroenterol 2006; 12(38): 6102-6108.

5. Burisch J, Jess T, Martinato M, et al. The burden of inflammatory bowel disease in Europe. J Crohns Colitis 2013; 7(4): 322-337.

6. Tursi A, Elisei W and Picchio M. Incidence and prevalence of inflammatory bowel diseases in gastroenterology primary care setting. Eur J Intern Med 2013; 24(8): 852-856. 
7. Di Domenicantonio R, Cappai G, Arcà M, et al. Occurrence of inflammatory bowel disease in central Italy: a study based on health information systems. Dig Liver Dis 2014; 46(9): 777-782.

8. Istituto di Ricerca della Società Italiana di Medicina Generale. VII Report Health Search, 2011-2012. https:// www.healthsearch.it/documenti/Archivio/Report /VIIReport_2011-2012/uniflip_publication/index.html (accessed December 18, 2018).

9. Gibson PR, Vaizey C, Black CM, et al. Relationship between disease severity and quality of life and assessment of health care utilization and cost for ulcerative colitis in Australia: a cross-sectional, observational study. J Crohns Colitis 2014; 8(7): 598-606.

10. Taft TH, Keefer L, Leonhard C, et al. Impact of perceived stigma on inflammatory bowel disease patient outcomes. Inflamm Bowel Dis 2009; 15(8): 1224-1232.

11. Cohen RD, Yu AP, Wu EQ, et al. Systematic review: the costs of ulcerative colitis in Western countries. Aliment Pharmacol Ther 2010; 31(7): 693-707.

12. Kawalec P. Indirect costs of inflammatory bowel diseases: Crohn's disease and ulcerative colitis. A systematic review. Arch Med Sci 2016; 12(2): 295-302.

13. Ordás I, Eckmann L, Talamini M, et al. Ulcerative colitis. Lancet 2012; 380(9853): 1606-1619.

14. Rutgeerts P, Sandborn WJ, Feagan BG, et al. Infliximab for induction and maintenance therapy for ulcerative colitis. $N$ Engl J Med 2005; 353(23): 2462-2476.

15. Reinisch W, Sandborn WJ, Hommes DW, et al. Adalimumab for induction of clinical remission in moderately to severely active ulcerative colitis: results of a randomised controlled trial. Gut 2011; 60(6): 780-787.

16. Sandborn WJ, Feagan BG, Marano C, et al. Subcutaneous Golimumab Induces Clinical Response and Remission in Patients with Moderate-to-Severe Ulcerative Colitis. Gastroenterology 2014; 146(1): 85-95; quiz e 14-15.

17. Sandborn WJ, Feagan BG, Marano C, et al. Subcutaneous Golimumab Maintains Clinical Response in Patients with Moderate-to-Severe Ulcerative Colitis. Gastroenterology 2014; 146: 96-109.

18. Feagan BG, Rutgeerts P, Sands BE, et al. Vedolizumab as induction and maintenance therapy for ulcerative colitis. $N$ Engl J Med 2013; 369(8): 699-710.

19. Feagan BG, Rubin DT, Danese S, et al. Efficacy of vedolizumab induction and maintenance therapy in patients with ulcerative colitis, regardless of prior exposure to tumor necrosis factor antagonists. Clin Gastroenterol Hepatol 2017; 15(2): 229-239.

20. Danese S, Fiorino G, Peyrin-Biroulet L, et al. Biological agents for moderately to severely active ulcerative colitis: a systematic review and network meta-analysis. Ann Intern Med 2014; 160(10): 704-711.

21. Vickers AD, Ainsworth C, Mody R, et al. Systematic review with network meta-analysis: comparative efficacy of biologics in the treatment of moderately to severely active ulcerative colitis. PLoS One 2016; 11(10): e0165435.

22. Jansen J, Mody R, Ursan I, et al. 3 Cost per clinical outcomes with biologics for the treatment of moderately to severely active ulcerative colitis. Gastroenterology 2015; 148(4) (Suppl 1): S-1-S-2.

23. Dias S, Sutton AJ, Ades AE, et al. Evidence synthesis for decision making 2: a generalized linear modeling framework for pairwise and network meta-analysis of randomized controlled trials. Med Decis Making 2013; 33(5): 607-617.

24. Sandborn WJ, van Assche G, Reinisch W, et al. Adalimumab induces and maintains clinical remission in patients with moderate-to-severe ulcerative colitis. Gastroenterology 2012; 142(2): 257-265.e1-3.

25. Laupacis A, Sackett DL and Roberts RS. An assessment of clinically useful measures of the consequences of treatment. N Engl J Med 1988; 318(26): 1728-1733.

26. Ruggeri M, Basile M, Armuzzi A, et al. Activity-based costing and budget analysis of vedolizumab versus conventional treatments in ulcerative colitis and Crohn's disease. GRHTA 2017; 4(1): e88-e99

27. European Medicines Agency. Adalimumab - Summary of Product Characteristics. http://www.ema.europa.eu/docs /en_GB/document_library/EPAR_-_Product_Information /human/000481/WC 500050870.pdf (accessed December 18, 2018).

28. Agenzia Italiana dal Farmaco. Adalimumab. Determina del 29.10.2007. GU n. 271 del 21-11-2007 - Suppl. Ordinario n. 239.

29. European Medicines Agency. Golimumab - Summary of Product Characteristics. http://www.ema.europa.eu/docs /en_GB/document_library/EPAR___Product_Information /human/000992/WC500052368.pdf (accessed December 18, 2018)

30. Agenzia Italiana dal Farmaco. Golimumab. Determina del 19.1.2015. GU n. 22 del 28-1-2015.

31. European Medicines Agency. Infliximab - Summary of Product Characteristics. http://www.ema.europa.eu /docs/en_GB/document_library/EPAR___Summary_for the public/human/000240/WC500050888.pdf (accessed December 18, 2018).

32. Ministero della Salute. Infliximab. Decreto del 10.4.2001. GU n. 101 del 3-5-2001.

33. European Medicines Agency. Vedolizumab - EPAR Summary for the Public. http://www.ema.europa.eu /docs/en_GB/document_library/EPAR_-_Summary_for the public/human/002782/WC500168532.pdf (accessed December 18, 2018).

34. Agenzia Italiana dal Farmaco.Vedolizumab. Determina del 31.3.2016. GU n. 87 del 14-4-2016.

35. Ross TK. Analyzing health care operations using ABC. $J$ Health Care Finance 2004; 30(3): 1-20.

36. Bruschi C. Case history: il controllo dei costi in sanità attraverso l'ABC - Activity Based Costing. (2015) http:// www.soluzionidimpresa.it/case-history-il-controllo-dei -costi-in-sanita-attraverso-l-abc-activity-based-costing/ (accessed December 18, 2018).

37. Wilson MR, Bergman A, Chevrou-Severac H, et al. Costeffectiveness of vedolizumab compared with infliximab, adalimumab, and golimumab in patients with ulcerative colitis in the United Kingdom. Eur J Health Econ 2018; 19(2): 229-240.

38. Toor K, Druyts E, Jansen JP, et al. Cost per remission and cost per response with infliximab, adalimumab, and golimumab for the treatment of moderately-to-severely active ulcerative colitis. J Med Econ 2015; 18(6): 437-446.

39. van der Valk ME, Mangen MJ, Severs M, et al. Evolution of costs of inflammatory bowel disease over two years of follow-up. PLoS One 2016; 11(4): e0142481. 